\title{
La dette écologique : d'une notion politique à un concept philosophique?
}

The ecological debt: from a political notion to a philosophical concept?

\section{Delphine Pouchain}

\section{(2) OpenEdition}

1 Journals

\section{Édition électronique}

URL : http://journals.openedition.org/developpementdurable/10208

DOI : 10.4000/developpementdurable.10208

ISSN : 1772-9971

Éditeur

Association DD\&T

Référence électronique

Delphine Pouchain, «La dette écologique : d'une notion politique à un concept philosophique? », Développement durable et territoires [En ligne], Vol. 5, n 1 | Février 2014, mis en ligne le 04 février 2014 consulté le 19 avril 2019. URL : http://journals.openedition.org/developpementdurable/10208 ; DOI : 10.4000/developpementdurable.10208

Ce document a été généré automatiquement le 19 avril 2019.

Développement Durable et Territoires est mis à disposition selon les termes de la licence Creative Commons Attribution - Pas d'Utilisation Commerciale 4.0 International. 


\title{
La dette écologique : d'une notion politique à un concept philosophique?
}

The ecological debt: from a political notion to a philosophical concept?

\author{
Delphine Pouchain
}

1 La notion de dette écologique, apparue dans les années 1990, revêt de nombreuses dimensions. Premièrement, dans sa dimension spatiale, la plus courante, la dette écologique renvoie à la dette due aux pays pauvres par les pays riches. Les pays riches se seraient développés en pillant ou en sous-payant les ressources naturelles des pays en développement, nous leur sommes donc redevables. C'est d'abord avec cette signification que la notion émerge, avant de voir son usage s'élargir. Deuxièmement, dans sa dimension temporelle, la notion renvoie à l'idée d'une dette due aux générations présentes par les générations passées. En ce sens, les générations passées sont alors responsables de leur mauvaise gestion des ressources naturelles et de la surexploitations de celles-ci. La dette écologique est alors un «(...) concept que l'on peut définir empiriquement comme l'ensemble des atteintes aux milieux naturels exercées par des humains dans le passé»" (Delord, Sébastien, 2010:1). Alors que le développement durable met en avant notre responsabilité à l'égard des générations futures, on s'intéresse ici à la dette des générations passées à notre égard. Si la logique est en partie différente, les mêmes problématiques sont parfois rencontrées. Bien-sûr, la dette due aujourd'hui aux pays pauvres revêt à la fois une dimension spatiale et temporelle, si l'on considère que les générations passées et les générations actuelles des pays du Nord sont redevables à l'égard des pays pauvres. Enfin, la dette écologique renvoie également à l'idée d'une dette que les hommes ont accumulé à l'égard de la nature, comme peut le révéler par exemple l'évolution de notre empreinte écologique. La notion de dette écologique interroge donc notre rapport à la nature (éthique environnementale), notre rapport aux pays en développement (justice sociale), et nos rapports aux générations passées et futures (justice intergénérationnelle). On peut également préciser que la notion de dette renvoie habituellement à une "[...] dette intentionnelle, formalisée généralement par une promesse 
contractuelle ex ante» (Delord, Sébastien, $2010: 6$ ). Ici, il s'agit a contrario d'une dette " non-intentionnelle qui surgit ex post, comme une différence dans les bilans, un ajustement afin de solder tout rapport créancier-débiteur.» (Delord, Sébastien, 2010: 6). Il paraît donc intéressant d'analyser cette notion polysémique, et d'examiner sa portée conceptuelle. S'agit-il d'une notion politique uniquement ou sommes-nous face à un véritable concept philosophique à même d'éclairer des enjeux de justice sociale et environnementale ? Audelà de son exploitation politique, quelle est la véritable portée de cette notion? Pour répondre à ces questions, nous rappellerons tout d'abord comment a émergé la notion d'abord politique de dette écologique. Dans un second temps, nous nous interrogerons sur le sens et les conséquences de l'extension du sens de cette notion. Enfin, nous nous demanderons si nous ne sommes pas face à une aporie philosophique au vu des questions que la notion fait émerger.

\section{L'irruption d'une notion politique}

\subsection{La mise en évidence de la dette écologique des pays riches}

2 La « dette écologique » renvoie initialement à la dette des pays riches à l'égard des pays pauvres. La notion de « dette écologique » a pour origine une idée très simple : «(...) la majorité qui surexploite les ressources naturelles (particulièrement les pays riches) est en situation de dette "écologique» aux dépens de ceux en possession de ces ressources (les pays pauvres)." (Delord, Sébastien, 2010:2). Cette notion a été forgée par une ONG chilienne (Instituto de Ecologia Politica) en 1990, avant d'être largement reprise par d'autres ONG d'abord, puis par les institutions internationales. On distingue généralement au sein de cette dette écologique plusieurs composantes, notamment la dette carbone, la biopiraterie, ou encore la dette due en raison du pillage des ressources naturelles des pays pauvres. Se pose biensûr immédiatement la question de l'évaluation de cette dette écologique. Selon MartinezAlier [2007], l'important à cet égard est de se demander si les conflits écologiques peuvent s'exprimer « à l'intérieur d'un seul standard d'évaluation » ou « à travers un conflit ou un désaccord sur un standard d'évaluation. » (Martinez-Alier, 2007 : 204). Le deuxième cas de figure apparaît lorsque sont en jeu des pertes irréversibles, au niveau de la biodiversité par exemple. Le plus souvent selon lui, «Différents langages d'évaluation sont utilisés. Les personnes démunies et dont la vie et la santé n'ont guère de valeur, en appellent souvent à des langages d'évaluation non chrématistiques. Qui a alors le pouvoir de simplifier cette complexité en imposant un seul langage d'évaluation?» (Martinez-Alier, 2007 : 204), sous-entendu ici le langage monétaire? En même temps, de nombreuses associations estiment que l'évaluation monétaire est indispensable, compte tenu du fait que cela serait le seul «langage " compréhensible et audible par les institutions internationales. Tout en reconnaissant que certains éléments de la dette sont sans doute d'une valeur infinie et donc inestimables, il leur paraît nécessaire de chiffrer un maximum de composantes au sein de la dette écologique. Cette dette est mise en évidence notamment via l'empreinte écologique. Si notre empreinte écologique dépasse l'empreinte écologique soutenable, c'est bien que nous sommes en situation de dette vis à vis de la nature. Par ailleurs, les différences d'empreinte écologique entre pays illustrent la dimension spatiale de la dette écologique. Pour visualiser cette dette, on peut aussi observer, chaque année, la date à partir de laquelle la consommation de ressources dépasserait la capacité de renouvellement de la planète. C'est ce que calcule la New Economics Foundation (NEF). 
Cette date est appelée « Jour de la dette écologique » ou « Jour du dépassement ». À partir de cette date, l'humanité creuse un peu plus sa dette écologique. D'après les calculs de la $\mathrm{NEF}$, cette date anniversaire se produit de plus en plus tôt chaque année. Cela signifie que notre stock de ressources disponible pour une année est épuisé de plus en plus rapidement. En 2013, le «jour du dépassement » était le 20 août. En 1987, le «jour du dépassement » se produisait en décembre, en 1995 en novembre. Cela signifie donc que nous mettons de moins en moins de temps chaque année pour consommer notre stock de ressources naturelles annuelles. Enfin, peuvent être mobilisés les travaux sur la valeur de l'environnement, qui tentent de calculer la «valeur économique totale» de l'environnement, en ajoutant ses valeurs d'usage, d'option, de legs et d'existence. Les méthodes directes et indirectes d'évaluation de l'environnement peuvent en effet fournir des indications quant au coût que représentent telles ou telles pollution ou destruction de ressources naturelles. Évidemment, l'évaluation monétaire de la dette écologique bute sur des problèmes rédhibitoires, du moins pour certaines composantes de cette dette dont le remboursement ne pourra plus jamais avoir lieu, nous y reviendrons. Il faut en effet garder en mémoire «(...) les paramètres d'incertitude, les impacts environnementaux noncomparables, la substituabilité limitée entre capital naturel et capital humain, le caractère arbitraire des taux d'escompte ainsi que les barrières éthiques, tel que le caractère sacré de la nature pour certains peuples. » (Delord, Sébastien, 2010: 4). Bref, autant d'obstacles qui rendent extrêmement problématique l'évaluation, monétaire ou non, de cette dette écologique.

\subsection{Dette écologique et « échange écologiquement inégal »}

3 La dette écologique est le produit de l'«échange écologiquement inégal » telle que l'expose l'économiste espagnol Martinez-Alier. Le concept d'« échange écologiquement inégal » signale surtout le fait que les produits sont exportés par les pays pauvres à des prix qui ne couvrent pas les coûts sociaux et environnementaux induits par leur production. Plus précisément, Martinez-Alier définit l'échange écologiquement inégal comme «le fait d'exporter des produits des régions et pays pauvres à des prix qui ne tiennent pas compte des externalités locales causées par ces exportations ou par l'épuisement des ressources naturelles en échange de biens et de services en provenance des pays riches» (Cité par Flipo, 2009: 65). Au-delà de la question plus traditionnelle de la dégradation des termes de l'échange et de l'échange inégal, on intègre ici la thématique des externalités négatives supportées par les pays pauvres, car non-rémunérées ou sous-évaluées. En effet, "En s'inspirant du concept marxien d'échange inégal, Martinez-Alier défend l'idée que les biens vendus par les pays du Sud, dans le cadre des marchés internationaux, subissent des prix "écologiquement incorrects". Et il en est ainsi parce que ces prix n'intègrent pas l'ensemble des coûts de dommages, liés aux productions dans ces pays, et spécialement les coûts de dommages environnementaux. Il se forme ainsi une "dette écologique" des pays du Nord vis-à-vis du Sud qui, dans une perspective d'équité internationale, devrait être prise en compte et faire contrepoids à la dette monétaire des pays les plus pauvres. » (Zuindeau, 2005 : 9). La notion d'« échange écologiquement inégal » a ainsi partie liée avec la notion de prix juste. Dans cette perspective, le prix juste, défini comme corrigeant les externalités négatives, peut être mis au service du développement durable. Dans cette conception du prix juste, on retrouve l'idée en plein développement actuellement d'une " vérité écologique » des prix. Il y a donc un lien entre prix juste et extinction de la dette : c'est quand le prix est juste que les agents économiques seraient 
véritablement quittes. À l'inverse, si le prix est injuste, il peut y avoir persistance d'externalités et donc dette.

\subsection{Dette écologique contre dette financière}

4 En 1992, est signé au sommet de la Terre de Rio de Janeiro un "Traité de la Dette ». Ce document relie directement la dette financière que les pays pauvres doivent aux pays riches et la dette écologique, dont les débiteurs seraient les pays riches et les créditeurs les pays pauvres. Ce Traité «(...) met l'accent sur la reconnaissance de la dette écologique du Nord envers le Sud ainsi que sur la nécessité d'annuler la dette extérieure du Sud envers le Nord. L'idée de relier dette écologique et dette extérieure est basée sur l'objectif d'instituer la première en valeur compensatoire afin de réduire voire d'éliminer la seconde. " (Delord, Sébastien, $2010: 3$ ). En 1999, à Johannesbourg, est lancée la «Campagne internationale pour la reconnaissance et la réclamation de la dette écologique ». En 2000, à Prague, des ONG en provenance de pays du sud se réunissent et forment «l'Alliance des peuples du Sud créanciers de la dette écologique ». En 2003 le Réseau européen pour la reconnaissance de la dette écologique voit le jour l'ENRED (European Network on Recognition of Ecological Debt). Si son montant fait débat compte tenu des nombreux problèmes d'évaluation, elle serait largement supérieure à la dette financière.

\section{Extension et dilution de la notion}

5 On peut schématiquement distinguer cinq types de dette au sein de la dette écologique : la dette écologique écocentrée ou biocentrée, et, au sein de la dette écologique anthropocentrée, la dette intragénérationnelle, intergénérationnelle et enfin transgénérationnelle. On peut alors observer qu'en fonction du type de «dette» écologique, la notion de dette revêtira une pertinence plus ou moins avérée.

\subsection{La dette écologique écocentrée ou biocentrée}

Lorsque l'on se demande "Qui doit quoi à qui », « la première réponse qui vient à l'esprit n'est autre que la nature elle-même! C'est à la Terre, à sa biodiversité, à son climat, à son sol et à son sous-sol, que nous sommes en train d'emprunter plus que de raison et que nous causons des préjudices irréversibles qu'il nous faudra dédommager.» (Delord, Sébastien, 2010: 6). Dans cette perspective, qui n'est pas celle des origines, la dette écologique renvoie prioritairement à nos devoirs vis-à-vis de la nature. Au sein de l'éthique environnementale, on distingue les éthiques écocentriques et biocentriques. Pour l'éthique biocentrique, la condition de vivant suffit à justifier le " respect de la nature " (Taylor, 1981). Paul Taylor (1986) défend dans cette logique l'idée d'une «justice restitutive", qui met en avant la nécessité de réparer les injustices faites à des organismes individuels, soit un moyen de rembourser notre dette. Il y a une «considérabilité morale » du vivant (Goodpaster, 1978), et ce indépendamment d'un critère souvent cité de sensibilité, de plaisir et de peine que peuvent ressentir certains êtres vivants. L'éthique écocentrique donne pour sa part la priorité au respect de l'écosystème. Une vie en moins n'est pas forcément dommageable si cela ne met pas en péril la pérennité de l'écosystème considéré. Comme le dit Aldo Leopold, "Une chose est juste lorsqu'elle tend à préserver l'intégrité, la stabilité et la beauté de la communauté biotique. Elle 
est injuste lorsqu'elle tend à l'inverse.» (Cité in Goodpaster, 2007 :61). Ces deux perspectives impliquent de revoir nos devoirs à l'égard de la nature, voire de défendre un extensionnisme légal, qui vise à transposer aux entités naturelles le concept de droits de l'homme (voir par exemple Stone, 1972). Dans tous les cas, évoquer cette dette à l'égard de la nature que nous devrions et pourrions rembourser suggère que «(...) l'humanité peut restaurer ou remettre à neuf l'environnement naturel» (Katz, 2007 : 349). Or, ce projet de restauration de la nature est selon Katz [2007] un énorme mensonge. Ce projet d'une restauration de la nature sert surtout d'après lui à soulager notre mauvaise conscience, et à nous persuader nous-mêmes que les dommages que nous occasionnons à la nature sont réparables et ne sont pas irréversibles. L'auteur critique par conséquent la notion de « justice restitutive » défendue par Taylor (1986) qui serait une chimère. Katz prend donc comme cible "(...) l'idée optimiste selon laquelle l'humanité aurait l'obligation et la capacité de réparer ou de reconstruire les systèmes naturels endommagés.» (Katz, 2007: 352). Cette possibilité de restauration n'est qu'un mythe, car une nature restaurée ne sera toujours qu'un artefact créé par les hommes pour répondre aux besoins d'autres hommes. Ils sont intrinsèquement anthropocentriques. Katz se déclare donc indigné «(...) par l'idée que l'on puisse nous faire prendre pour une "nature" qui serait le fruit de la technologie pour la réalité." (Katz, 2007: 355). Quoique nous fassions, "Nous ne restaurons pas la nature; nous ne la remettons pas à neuf. La restauration de la nature est un compromis; elle ne devrait pas être une mesure fondamentale de politique environnementale.» (Katz, 2007 : 371). Bref, la meilleure volonté du monde ne l'empêchera jamais de n'être qu'un second best. Comme le dit autrement Jonas (1990), une nature humanisée est intrinsèquement une nature aliénée. En s'appuyant sur la logique développée par Katz, on peut dire qu'on est en présence ici d'une dette qui tend vers le don, puisque cette dette paraît largement non-remboursable ${ }^{1}$ : «(...) cette dette écologique écocentrée nous rappelle que nous sommes les fruits d'une nature qui nous fait en permanence don d'elle-même. Originairement, et de multiples mythologies issues des peuples anciens de la Terre sont là pour le célébrer, nous sommes en perpétuelle situation de dette, de dépendance et de fragilité vis-à-vis de la nature. Nous l'avions oublié, nous les peuples riches de l'Occident; le surendettement conjoncturel qui nous affecte est là pour nous le remémorer plus que jamais. » (Delord, Sébastien, $2010:$ 7). Comme le dit joliment Serres, «(...) rien de beau ne se produit sans ce donateur gracieux de toutes les magnificences. " (Serres, 1992: 45). Dans sa logique d'un contrat naturel, il importe de reconnaître ce que la nature donne à l'homme, et «(...) celui-ci doit rendre à celle-là, devenue sujet de droit.» (Serres, 1992: 67, souligné par nous). Il évoque un "programme des restitutions» (Serres, 1992:68) qui rappelle bien l'idée de dette. En effet, selon Serres, chaque partenaire doit au minimum à l'autre la vie. Selon lui, "Le monde, globalement, et les phénomènes, prochains, locaux ou lointains, nous sont donnés; il y aurait de l'injustice, un déséquilibre, à ce que nous recevions ce donné gratuitement, sans jamais rendre quoi que ce soit. L'équité donc veut que nous rendions, au moins autant que nous recevons, c'est à dire suffisamment.» (Serres, 1992 : 141). À la question de savoir ce que nous pouvons rendre au monde qui nous donne, Serres répond la raison, réponse à travers laquelle il faut comprendre un usage raisonné du monde. Ainsi, contrairement à ce suggère Juvin, la dette écologique, que l'on devrait donc nommer don, nous signale que tout ne peut être produit. Si l'on peut le suivre dans l'idée d'un don qui a été gaspillé ou mal géré par les hommes, cela ne signifie pas pour autant que la production humaine pourra se substituer au don de la nature. Pour ce dernier, «(...) il s'agit d'inventer les modes de fabrication d'un environnement qui ne nous est plus donné. Plus question d'exploiter et de prélever, il faut inventer et produire.» (Juvin, 2008: 217). Ici, la production humaine remplace la dette et enterre le don. Parler de dette à l'égard de la 
nature n'a pas de sens dans la mesure où nous deviendrons capables de "produire la nature ». Cependant, cette première conception de la dette écologique reste insuffisante, car elle néglige les inégalités environnementales et fait de l'humanité une catégorie homogène dans ses rapports avec la nature. Cette approche, qui ignore le point de vue de la justice environnementale, doit être complétée par une dette écologique anthropocentrée.

\subsection{Les dettes écologiques anthropocentrées}

7 La reconnaissance d'une dette écologique non-anthropocentrée ne doit pas faire oublier que les responsabilités ne sont pas également partagées, et que la dette écologique renvoie aussi à l'idée d'une dette anthropocentrée, qui peut être intragénérationnelle ou intergénérationnelle. La dette intragénérationnelle s'observe lorsque c'est la même génération qui a contracté la dette et qui a la possibilité de la rembourser. C'est la dette par exemple que la génération actuelle des pays riches doit aux habitants actuels des pays pauvres. La dette intergénérationnelle renvoie au cas de figure de la succession de générations au sein d'un même groupe. Le terme de dette peut sembler ici une nouvelle fois impropre. L'existence de cette dette peut être remise en cause en mobilisant Solow (1974). En effet, ses arguments semblent récuser l'idée même d'une telle dette. Il ne s'agirait alors pas d'une dette, mais bien d'un simple échange entre les générations. Les générations passées ont puisé dans le stock de capital naturel, mais elles nous ont légué en échange davantage de capital physique, donc nous sommes quittes. Si le stock de capital global est resté inchangé, alors il y a bien eu équivalence. Les générations passées ne nous doivent rien. Enfin, on évoque parfois également une dette transgénérationnelle qui combine ces deux dimensions. C'est typiquement le cas de la dette due aux pays pauvres, à la fois par les générations passées et actuelles des pays riches.

\section{Une aporie philosophique?}

8 La notion de dette écologique pose quatre questions qui sont autant d'obstacles potentiels à la constitution d'un véritable concept philosophique.

\subsection{Comment sanctionner les générations passées?}

9 La question est posée par Gosseries: "Chaque génération est-elle en mesure de proférer à l'égard de celle(s) avec qui elle est supposée coopérer des menaces crédibles en cas de violation des règles de justice intergénérationnelle?» (Gosseries, 2010 : 199). La sanction des générations passées suppose, sauf dans le cas d'une sanction purement symbolique, que ces générations soient encore présentes, et qu'il y ait donc un réel enchevêtrement des générations : "Les menaces descendantes - à savoir envers la génération suivante, si elle ne respecte pas ses obligations envers la génération de ses parents - sont envisageables en cas d'enchevêtrement intergénérationnel. En revanche, les menaces ascendantes - d'une génération envers celle qui la précède si elle ne respecte pas ses obligations descendantes - sont nettement moins crédibles. C'est là que se situe la difficulté la plus sérieuse. L'enchevêtrement est absolument nécessaire ici, en particulier parce que la sanction ne peut intervenir qu'après constatation de la violation éventuelle de la règle intergénérationnelle. Comment une génération pourrait-elle sanctionner une génération précédente, si cette dernière n'existe plus?» (Gosseries, 2010 : 199). 
Un autre problème apparaît et s'ajoute au premier, à savoir le fait que les générations actuelles pourraient pâtir de sanctions imposées aux générations passées, dans la mesure où l'héritage risque alors d'être moindre: "En outre, si l'on accepte l'idée selon laquelle ce dont nous héritons nous arrive entièrement par le canal de la génération précédente, sanctionner cette dernière, c'est s'exposer à recevoir moins soi-même si la sanction prend une forme minimalement patrimoniale. Hormis le cas d'une sanction symbolique, toute sanction ascendante équivaudrait en fait alors à une auto-sanction pour la génération même qui l'édicte. Elle se tirerait une balle dans le pied.» (Gosseries, 2010 : 199). C'est donc in fine la génération actuelle qui se retrouve également sanctionnée.

\subsection{Pouvons-nous être redevables des dettes octroyées par autrui ?}

10 La deuxième objection concerne ce que Gosseries appelle "l'exemption d'impuissance »: "Une personne ne saurait être tenue pour moralement responsable des conséquences dommageables de l'action d'autrui si elle n'était pas en mesure (physiquement) de s'opposer de quelque manière que ce soit à un tel acte." (Gosseries, 2003: 11). S'il n'y a pas enchevêtrement des générations, et que les générations passées ne sont plus là pour régler leurs dettes, peut-on envisager que les générations actuelles remboursent la dette des générations passées ? Le problème, c'est que " (...) nul ne peut être tenu pour responsable des actes d'autrui auxquels il n'est pas en mesure de s'opposer (en effet, ils ont déjà eu lieu). Conséquence: la dimension historique de la crise actuelle n'a pas à être prise en compte, elle n'intervient que comme un donné de la situation présente. Les actes des générations passées doivent être traités comme des événements naturels. ( (Larrère, 2009: 159-160). En même temps, d'après Larrère, "On peut juger que cette façon de naturaliser la situation relève de la mauvaise foi des avocats des pays du Nord. Peut-être les générations présentes n'avaient-elles pas les moyens de s'opposer à l'ensemble des décisions qui ont mis en cuvre la révolution industrielle, mais elles en sont largement bénéficiaires. Accepter un héritage, n'est-ce pas du même coup accepter la responsabilité de ses conséquences (bonnes et mauvaises)?» (Larrère, 2009: 159-160). D'un côté, certes, on ne peut être tenu responsable des manquements des générations passées. En même temps, les générations actuelles bénéficient largement de cet héritage que leur ont transmis les générations passées : «Les Français de la génération actuelle profitent d'investissements et d'activités de la génération précédente rendus possibles par les émissions passées de $\mathrm{CO}_{2^{*}}$ (...) à Cette approche fondée sur une notion quelque peu transformée de free riding est possible. Reste à déterminer précisément ce que le free rider devrait moralement en retour.» (Gosseries, 2006: 43). En ne remboursant pas la dette des générations passées, notre génération se conduirait en passager clandestin. Il pourrait donc être légitime de faire payer les générations actuelles pour les erreurs commises par les générations antérieures ${ }^{2}$. L'exemption d'impuissance est alors reformulée ainsi : «Une personne ne saurait être tenue pour moralement responsable des conséquences dommageables de l'action d'autrui si elle n'était pas en mesure (physiquement) de s'opposer de quelque manière que ce soit à un tel acte. Néanmoins, elle peut malgré tout être tenue à compensation pour de telles conséquences dommageables sur autrui si et seulement si, une fois que ces dernières furent ou auraient dû être mises en lumière $\left(t_{c}\right)$, elle en tirait encore des bénéfices. » (Gosseries, 2003 : 12). 


\subsection{Suffit-il de transmettre une dette équivalente?}

11 Est-il moralement suffisant de ne pas transmettre une dette plus élevée que celle que nous avons reçue? Gosseries défend en effet cette idée, qu'il appelle principe de "l'interdiction de désépargne ». Cela signifie que chaque génération doit "seulement " effacer les conséquences négatives de ses actes. Pour le dire autrement, le stock de la dette à transmettre doit demeurer inchangé. On retrouve, présentée autrement, une logique qui rappelle celle de Solow évoquée précédemment: puisque le montant de l'héritage doit rester identique, cela signifie bien que le stock de capital disponible doit rester constant. Néanmoins, à la différence de Solow, Gosseries envisage l'éventualité d'une " clause de naturalité » selon laquelle " (...) un pourcentage donné du capital physique transmis devrait être constitué d'espaces naturels. » (Gosseries, 2004 : 248-249). À rebours de la position rawlsienne, le stock de capital ne doit pas non plus augmenter : l'interdiction de désépargne est également une interdiction d'épargne. Ainsi, «(...) une fois passée une courte phase d'accumulation, l'égalitariste devrait donc prôner un refus de la désépargne générationnelle (qui consisterait à transférer moins que ce que l'on a hérité) mais aussi une interdiction de l'épargne générationnelle (qui consisterait à transférer plus à la génération suivante que ce dont on a hérité) (...). Il devrait le faire car ce dont nous disposons en sus par rapport à ce que nous avons hérité devrait plutôt bénéficier aux plus défavorisés au sein de notre génération. (Gosseries, 2010 : 197). En effet, épargner, et transmettre une dette inférieure à celle que l'on a reçue, signifie privilégier les générations futures aux membres les plus défavorisés de la génération actuelle. Autrement dit, si nous avions les ressources pour réduire le stock de la dette, dans une perspective ralwsienne, il faudrait utiliser ces moyens supplémentaires pour améliorer le sort des plus défavorisés dès aujourd'hui. La seule exception à l'obligation d'un taux d'épargne nul, et donc le seul argument acceptable pour une augmentation du stock trouve sa source dans le "désavantage exogène anticipable » [Gosseries, 2002]. Dans ce cas, si la génération actuelle a les moyens d'anticiper une détérioration future de ce stock pour des motifs qu'elle ne peut contrôler, alors elle a le devoir de transférer à la génération à venir plus que ce dont elle a hérité. La règle est alors de transmettre une dette plus faible. Inversement, la génération actuelle est autorisée à puiser dans le stock de ressources et à désépargner si elle s'estime la victime innocente d'un désavantage exogène. Selon une variante plus normative, on peut aussi considérer que nous devons transférer à la génération suivante au moins autant que ce nous aurions dû recevoir de la part de la génération précédente. Précisons enfin que les principes d'interdiction de désépargne et d'épargne s'appliquent toutes choses égales par ailleurs démographiquement parlant. D'après Gosseries, un consensus se dégage donc sur la règle de l'interdiction de la désépargne, ce qui revient à dire que "(...) la génération actuelle a pour consigne d'effacer en quelque sorte toute conséquence négative de ses actes, de faire en sorte que le monde soit aussi bien que si elle n'avait jamais existé. Rien de moins, mais rien de plus.» (Gosseries, 2004 : 194).

\subsection{Quid de l'argument « les générations passées ne savaient pas»?}

12 Il s'agit de l'objection appelée « exemption d'ignorance » par Gosseries [2003], objection selon laquelle « une personne ne saurait être tenue pour moralement responsable des conséquences dommageables de ses propres actes si celles-ci n'étaient pas connues par 
elle et si elles n'auraient raisonnablement pas avoir dû être connues au moment où l'action eut lieu. » (Gosseries, $2003:$ 7). On peut faire remarquer effectivement qu'« Il va de soi que celui qui ne sait pas qu'il est en train de causer des dommages à l'environnement ne saurait être tenu moralement responsable de ces dommages. Si les personnes concernées l'avaient su à l'époque, elles auraient peut-être renoncé à l'utilisation de ces bombes aérosols ou en auraient au moins limité l'utilisation au strict minimum. Si nul n'est censé ignorer la loi morale qui nous oblige à préserver notre environnement naturel, on ne saurait attendre des hommes qu'ils connaissent à tout moment les effets de chacun de leur type d'actions sur l'environnement naturel." (Campagna, 2010: 7). Le même constat est fait par Delord et Sébastien: "Cette pensée traditionnelle de la promesse et de la dette est précisément au fondement d'une responsabilité entendue comme la réponse à un engagement tenu pour absolu et irrévocable car pris antérieurement et en toute connaissance de cause. Or, les générations passées, en s'engageant sur le chemin d'un endettement écologique massif, ne se sont rendu compte des effets de leurs actions qu'ultérieurement et n'ont pas agi en toute connaissance de cause. Selon la tradition, on devrait en conclure qu'elles ne sauraient être tenues responsables moralement ou pratiquement, car aucun engagement ne peut leur être assigné à un point fixe du passé.» (Delord, Sébastien, 2010 : 14). Le concept de dette écologique serait ainsi privé de sens, dans la mesure où "On ne peut attribuer la responsabilité du changement climatique aux générations passées des pays du Nord: en s'engageant dans la révolution industrielle, elles en ignoraient les conséquences. " (Larrère, 2009 : 159). On est ici face au problème lié à «(...) un excès de notre pouvoir de faire sur notre pouvoir de prévoir et sur notre pouvoir d'évaluer et de juger. (Jonas, 1990 : 58). Cependant, cette incohérence serait levée selon le philosophe Bernard Williams grâce à la notion de «fortune morale». L'idée générale est la suivante: «(...) l'issue imprévisible rétroagit sur le jugement que l'on porte sur la conduite de cet homme et aussi sur le jugement qu'il porte lui-même sur sa propre conduite.» (Dupuy, 2010). Dans son ouvrage de 1976 dont le titre a été traduit par $L a$ fortune morale, Williams explique que «Lorsque les conséquences d'une action que l'on envisage d'entreprendre sont grevées d'une très forte incertitude, que la nature de celle-ci interdit ou rend dérisoire le calcul probabiliste des conséquences, et qu'on ne puisse exclure une issue catastrophique, alors il n'est pas déraisonnable d'admettre que le jugement à porter sur l'action ne puisse être que rétrospectif - c'est-à-dire qu'il doive prendre en compte les événements postérieurs à l'action dont il était impossible de prévoir, même en probabilité, la survenue au moment d'agir.» (Dupuy, 2010). L'argument est repris par Dupuy, qui le résume ainsi: "(..) l'humanité prise comme sujet collectif a fait un choix de développement de ses capacités virtuelles qui la fait tomber sous la juridiction de la fortune morale. Il se peut que son choix mène à de grandes catastrophes irréversibles; il se peut qu'elle trouve les moyens de les éviter, de les contourner ou de les dépasser. Personne ne peut dire ce qu'il en sera. Le jugement ne pourra être que rétrospectif. Cependant, il est possible d'anticiper, non pas le jugement lui-même, mais le fait qu'il ne pourra être porté que sur la base de ce que l'on saura lorsque le voile de l'avenir sera levé.» (Dupuy, 2010). Ainsi, la dette peut faire son apparition beaucoup plus tard.

13 Aux confluents de la justice environnementale et de la justice sociale, la notion de dette écologique révèle la nécessité des réflexions en termes d'éthique environnementale et de justice intergénérationnelle. Si la dette écologique comme notion politique doit faire face à des problèmes d'évaluation aigus, la dette écologique comme concept philosophique soulève des questions embarrassantes. Si certaines d'entre elles semblaient faire de la notion de dette écologique une aporie philosophique, une réflexion plus poussée témoigne en réalité de la pertinence avérée de cette notion. 


\section{BIBLIOGRAPHIE}

Afeissa Hicham S., 2007, Éthique de l'environnement. Nature, valeur, respect, Paris, Vrin, textes clés d'éthique de l'environnement.

Campagna N., 2010, «L'agir respectueux de l'environnement comme but de la politique. », VertigO - La revue en sciences de l'environnement, vol. 10, $\mathrm{n}^{\circ}$ 1, avril. En ligne, http://

vertigo.revues.org/9446

Delord J., Sébastien L., 2010, « Pour une éthique de la dette écologique », VertigO - La revue en sciences de l'environnement, vol. 10, n 1, avril, en ligne sur http://vertigo.revues.org/9509

Dupuy J.-P., 2010, « Catastrophes et fortune morale », revue en ligne Hors-Sol, novembre, en ligne, http://hors-sol.net/revue/jean-pierre-dupuy-catastrophes-et-fortune-morale/

Flipo F., 2009, «Les inégalités écologiques et sociales : l'apport des théories de la justice », Mouvements, vol. 4, $\mathrm{n}^{\circ}$ 60, octobre-décembre, p. 59-76.

Goodpaster K. E., 1978, "On Being Morally Considerable", Journal of Philosophy, vol. 75, n 6, p. 308-325, traduit in Éthique de l'environnement. Nature, valeur, respect, 2007, Afeissa H.-S., Paris, Vrin, textes clés d'éthique de l'environnement.

Gosseries A., 2002, « La justice entre les générations. Faut-il renoncer au maximin intergénérationnel ?", Revue de métaphysique et de morale, vol. 1, n³3, p. 61-81.

Gosseries A., 2003, "Émissions historiques et free riding", Archives de philosophie du droit, vol. 47, pp. 191-220, 35 pages, en ligne http://www.uclouvain.be/cps/ucl/doc/etes/ documents/2003Emissionshist.pdf

Gosseries A., 2004, Penser la justice entre les générations : de l'affaire Perruche à la réforme des retraites, Paris, Edition Aubier, collection Alto.

Gosseries A., 2006, «Égalitarisme cosmopolite et effet de serre », Idées pour le débat, n 5.

Gosseries A., 2010, « Une métaphore de la justice intergénérationnelle », Regards croisés sur l'économie, vol. 1, n 7, p. 193-202.

Jonas H., 1990 (1979), Le principe responsabilité. Une éthique pour la civilisation technologique, Champs, Essai.

Juvin H., 2008, Produire le monde. Pour une croissance écologique, Gallimard Le Débat.

Katz E., 1990, "The Big Lie: Human Restoration of Nature", conférence prononcée à l'université de Columbia, lors d'un colloque consacré à la « Philosophie morale dans le domaine public », traduit in Éthique de l'environnement. Nature, valeur, respect, 2007, Afeissa H.-S., Paris, Vrin, textes clés d'éthique de l'environnement.

Larrère C., 2009, « La justice environnementale », Multitudes, vol. 1, n 36, p. 156-162.

Lestel D., 2010, L'animal est l'avenir de l'homme. Munitions pour ceux qui veulent (toujours) défendre les animaux, Fayard.

Martinez-Alier J., 2007, « Conflits de distribution écologique, identité et pouvoir », in Cornut P., Bauler T., Zaccai E., Environnement et inégalités sociales, Éditions de l'université de Bruxelles, Aménagement du territoire et environnement. 
Serres M., 1992 (1990), Le contrat naturel, Flammarion, Champs essai.

Solow R., 1974, « The Economics of resources or the Resources of Economics", The American Economic Review, vol. 64, n 2, p. 1-14.

Stone C. D., 1972, Should Trees Have Standing ? Towards Legal Rights for Natural Objects, Southern California LR.

Taylor P. W., 1981, "The Ethics of Respect of Nature", Environnemental Ethics, vol. 3, n 3, pp. 197-218, traduit in Éthique de l'environnement. Nature, valeur, respect, 2007, Afeissa H.-S., Vrin, textes clés d'éthique de l'environnement.

Taylor P., 1986, Respect for Nature, Princeton, Princeton University Press.

Zuindeau B., 2005, «Équité territoriale : quelles lectures par les théories du développement durable?», Reflets et perspectives de la vie économique, vol. 4 Tome XLIV, p. 5-18.

\section{NOTES}

1. Idée partagée par le philosophe Dominique Lestel, qui évoque "une dette infinie ", "(...) une dette un peu particulière, contractée à la fois dans le passé et dans le futur. Une dette qui a la caractéristique de ne jamais pouvoir être remboursée.» (Lestel, 2010 : 9). Prendre beaucoup implique donc selon lui de donner beaucoup. Selon lui, nous ne sommes humains que grâce à la nature, et plus particulièrement aux animaux, ce qui implique cette dette infinie vis-à-vis d'eux. Lestel explique que cette dette est infinie pour trois raisons : «(...) d'abord parce qu'elle plonge dans le passé immémorial de l'homme; ensuite parce qu'elle s'étend jusqu'au futur le plus lointain de l'homme; enfin parce qu'elle est sans fond. » (Lestel, 2010 : 132). Il ajoute enfin : "La dette que l'homme a contractée à l'égard de l'animal est d'une profondeur insondable. Elle est en effet infinie, parce que l'homme ne pourra jamais s'en acquitter de façon satisfaisante et qu'il doit organiser sa vie en remboursant en permanence cette dette constitutive et abyssale. L'une des dimensions de cette dette est donc de devoir être remboursée continuellement. » (Lestel, $2010: 133)$.

2. Voir pour plus de détail l'analyse de Gosseries, à partir de l'exemple très instructif du Bengladesh et des États-Unis : «La proposition selon laquelle les américains d'aujourd'hui sont des freeriders aux dépens des bangladeshi d'aujourd'hui est valide dans notre exemple hypothétique. Nous pensons qu'elle l'est aussi dans le monde réel, dans la mesure où les américains d'aujourd'hui bénéficient encore des conséquences des émissions effectuées par leurs ancêtres (...) et dans la mesure où de telles émissions passées ont aujourd'hui des conséquences négatives sur les habitants d'autres pays, y compris du Bengladesh. Nous pourrions aussi aisément défendre la position complémentaire selon laquelle, pour ce qui est des émissions actuelles, la génération américaine actuelle est aussi une parasite du Bengladesh. Les américains d'aujourd'hui sont ainsi des free-riders du Bengladesh pour ce qui est des émissions historiques et des parasites du Bengladesh pour ce qui est des émissions actuelles. Bien sûr, dans le monde réel, la même chose vaut aussi pour l'Europe envers le Bengladesh. (Gosseries, 2003, p. 17, souligné par nous). 


\section{RÉSUMÉS}

La notion de dette écologique émerge dans les années 1990. Il s'agit à l'origine de la dette que les pays développés doivent aux pays en développement. Elle servira rapidement d'argument pour annuler la dette financière des pays en développement. Se posera alors la question de son évaluation. Sa signification sera par la suite élargie, notamment dans le sens d'une dette que les hommes doivent à la nature. Malgré certaines interrogations semblant rédhibitoires, la notion n'en demeure pas moins un concept philosophique pertinent ou tout du moins stimulant.

The notion of ecological debt emerged in the 1990's. Originally, it is a matter of debt which developed countries owe to developing countries. It will quickly become an argument to cancel the financial debt of developing countries. Then the question of its evaluation will arise. Next its meaning will be widened, in particular in the sense of a debt that men owe to nature. Despite some questions which seems crippling, this notion remains a relevant philosophical concept, or at least a stimulating one.

\section{INDEX}

Keywords : debt, justice, environment, value, developing countries

Mots-clés : dette, justice, environnement, valeur, pays en développement

\section{AUTEUR}

\section{DELPHINE POUCHAIN}

Delphine Pouchain enseigne les sciences économiques à l'IEP de Lille et est membre du CLERSE. Elle a soutenu une thèse sur le commerce équitable et la notion de prix juste. Elle travaille plus généralement sur les questions d'histoire de la pensée économique, de philosophie économique et de justice. delphine.pouchain@sciencespo-lille.eu 